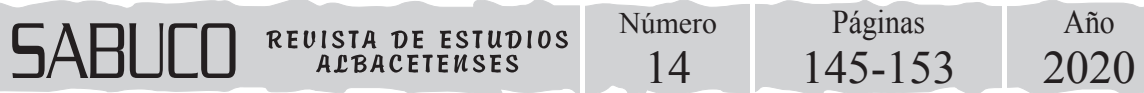

\section{DATOS SOBRE LA REPRODUCCIÓN DEL FLAMENCO COMÚN (PHOENICOPTERUS ROSEUS), EN LA LAGUNA DE PÉTROLA (ALBACETE, CASTILLA-LA MANCHA). PERIODO 2017-2020}

\section{DATA ON THE COMMON FLAMINGO REPRODUCTION (PHOENICOPTERUS ROSEUS), IN THE PÉTROLA LAGOON (ALBACETE, CASTILLA-LA MANCHA). PERIOD 2017-2020}

Por

Juan PICAZO TALAVERA ${ }^{1,2}$

1. Instituto de Estudios Albacetenses "Don Juan Manuel” de la Excma. Diputación de Albacete, Calle de las Monjas, s/n, 02005 Albacete.

2. Sociedad Albacetense de Ornitología. Apartado de correos n 18, 02080 Albacete. Autor para correspondencia: Juan Picazo picazotalavera@gmail.com

Cómo citar este artículo:

Picazo, J. (2020). Datos sobre la reproducción del flamenco común (Phoenicopterus roseus), en la laguna de Pétrola (Albacete, Castilla-La Mancha). Periodo 2017-2020. Sabuco, 14: 145-153

http://doi.org/10.37927/sabuco.14_6 



\section{RESUMEN}

Se aportan nuevos datos sobre la reproducción del flamenco común (Phoenicopterus roseus) en la laguna de Pétrola (Albacete, Castilla-La Mancha), correspondientes al periodo 2017-2020.

La especie se reprodujo en 2017, 2019 y 2020, alcanzando máximos históricos en Pétrola durante el primer año, en lo relativo al número de parejas (2600), pollos nacidos (2365) y pollos que volaron (1973). Durante 2019 se produjo un mínimo en el éxito de la reproducción, con un $3 \%$. De 400 parejas, nacieron sólo 18 pollos, de los cuales volaron 13 .

Palabras clave: Phoenicopterus roseus, laguna de Pétrola, reproducción, periodo 2017-2020.

\section{SUMMARY}

We are providing new data on the breeding of the Greater Flamingo (Phoenicopterus roseus) in Pétrola lagoon (Albacete, Castilla-La Mancha, SE Spain), during 2017-2020.

The highest number was recorded in 2017, when there were 2600 breeding pairs, 2365 hatched chicks and 1973 fledged chicks. Breeding success in 2009 was extremely low $3 \%$. Only 18 chicks were born out of 400 pairs, of which 13 flew.

Key word: Phoenicopterus roseus, Pétrola lagoon, breeding, period 20172020.

El flamenco común (Phoenicopterus roseus [Linnaeus, 1758]), es un ave acuática que actualmente tiene presencia habitual en los humedales del complejo lagunar de Pétrola-Corral Rubio-La Higuera (Picazo, 2011), en Albacete, comunidad autónoma de Castilla-La Mancha, en el sureste de la península Ibérica.

Su seguimiento en el citado complejo, realizado mensualmente desde 1998, ha permitido conocer datos básicos sobre la reproducción de la 
especie en la zona. Picazo (2018), ofreció información sobre 7 periodos de cría en la laguna de Pétrola hasta 2016. Aquí se completan los datos sobre su reproducción hasta la actualidad, es decir, entre 2017 y 2020 (ambos inclusive).

Para recabar datos sobre la reproducción del flamenco común en la laguna de Pétrola, se visitó el humedal de forma periódica entre abril (mayo en 2020) y septiembre; en 2017 cada 9,5 días, en 2018 no hubo cría, en 2019 cada 5,9 y en 2020 cada 5,5 jornadas. En el último caso, las visitas se iniciaron a partir de mediados de mayo debido a la imposibilidad de realizar trabajo de campo previamente, por las restricciones de movimiento derivadas del estado de alarma decretado por el Gobierno de España para combatir el virus SARS-CoV-2.

Durante las visitas y según los casos, se concretaron los siguientes parámetros relacionados con la reproducción: número de parejas, número de pollos nacidos y número de pollos que volaron (que fueron capaces de volar). Se estimaron los periodos de puesta, nacimiento de pollos y capacidad de vuelo.

El éxito de la reproducción anual se calcula en tanto por ciento, como el número de pollos que volaron, en relación con el número de parejas nidificantes. Cada pareja pone habitualmente sólo un huevo por intento de cría (Johnson y Cézilly, 2007).

La metodología utilizada para concretar numéricamente la población nidificante fue el recuento directo desde un lugar prominente (siempre el mismo), con buena visibilidad (distancia máxima de 600 metros; y diferencia de cota 17 metros). El horario y las condiciones meteorológicas fueron favorables para la observación. Se utilizó un catalejo con lentes de alta calidad y aumentos $\mathrm{x} 20$-x60. Posteriormente se realizaron análisis fotográficos en gabinete. Cuando el recuento preciso no fue posible, se hicieron estimas, indicadas con un asterisco $\left(^{*}\right)$ en los resultados. También se controlaron las reocupaciones, los huevos sin eclosionar y los cadáveres de pollos. Durante el año 2019 se accedió a la colonia una vez terminada la reproducción, haciéndose un recuento de nidos.

En las tablas 1 y 2 se ofrecen los datos obtenidos sobre parejas reproductoras, pollos y periodos de puesta, nacimiento de pollos y capacidad de vuelo. 


\begin{tabular}{ccccc}
\hline AÑN & $\begin{array}{c}\text { Parejas } \\
\text { reproductoras }\end{array}$ & $\begin{array}{c}\text { Pollos } \\
\text { nacidos }\end{array}$ & $\begin{array}{c}\text { Pollos que } \\
\text { volaron }\end{array}$ & $\begin{array}{c}\text { Exito de la } \\
\text { reproducción }\end{array}$ \\
\hline $\mathbf{2 0 1 7}$ & $2600 *$ & 2365 & 1973 & $76 \%$ \\
\hline $\mathbf{2 0 1 8}$ & --- & --- & -- & $3 \%$ \\
\hline $\mathbf{2 0 1 9}$ & $400 *$ & 18 & 13 & $82 \%$ \\
\hline $\mathbf{2 0 2 0}$ & $2050 *$ & $2000 *$ & 1680 & - \\
\hline
\end{tabular}

Tabla1.- Parámetros de la reproducción del flamenco común en la laguna de Pétrola, entre 2017 y 2020. Las cifras con asterisco* son estimas. En 2018 no hubo reproducción.

\begin{tabular}{cccc}
\hline AÑOS & Periodo de puesta & Nacimiento de pollos & Vuelo de pollos \\
\hline $\mathbf{2 0 1 7}$ & 30 abr-22 may & 1 jun-21 jun & 20 ago-15 sep \\
\hline $\mathbf{2 0 1 8}$ & --- & -- & -- \\
\hline $\mathbf{2 0 1 9}$ & 6 may-1 jun & 19 jun-1 jul & 7 sep-15(20) sep \\
\hline $\mathbf{2 0 2 0}$ & 23 abr-6 jun & 26 may-2 jul & 23 ago-25 sep \\
\hline
\end{tabular}

Tabla 2.- Periodos en los que ocurrieron la puesta, nacimiento y vuelo de pollos (capacidad de vuelo). Colonia del flamenco común en la laguna de Pétrola, entre 2017 y 2020. En 2018 no hubo reproducción.

Merecen especial mención los resultados obtenidos durante 2017 (Picazo, 2019), por alcanzarse máximos históricos en la colonia de Pétrola, en el número de parejas, pollos nacidos y pollos que volaron. Se trató de una temporada con niveles altos de agua en la generalidad de humedales del complejo desde febrero hasta junio (Picazo, inédito), lo que debió repercutir muy positivamente en las posibilidades alimentarias de las aves. El flamenco llegó a ocupar durante dicho periodo entre 19 y 23 humedales simultáneamente (Picazo, inédito).

En lo relativo al éxito de la reproducción, se produjo un mínimo histórico en 2019 (3 \%), inferior al 7 \% de 2015 (véase Picazo, 2018). Este bajo éxito estuvo motivado por un abandono generalizado de la colonia, 
del que desconocemos el motivo, aunque fue precedido por la entrada tardía de aves al complejo lagunar (figura 1) y el comienzo de la puesta a primeros de mayo, también algo tardía para la colonia de Pétrola (donde el inicio de la incubación ha ocurrido invariablemente durante abril; Picazo, 2018). Los niveles hídricos aumentaron significativamente entre abril y mayo (Picazo, inédito), previamente a la entrada de las aves (máximo en el complejo lagunar de 2493 flamencos durante el censo de mayo de 2019; Picazo, inédito), aunque posteriormente se produjo una disminución progresiva de niveles hídricos entre junio-agosto llegando a secarse bastantes lagunas durante los dos últimos meses citados.

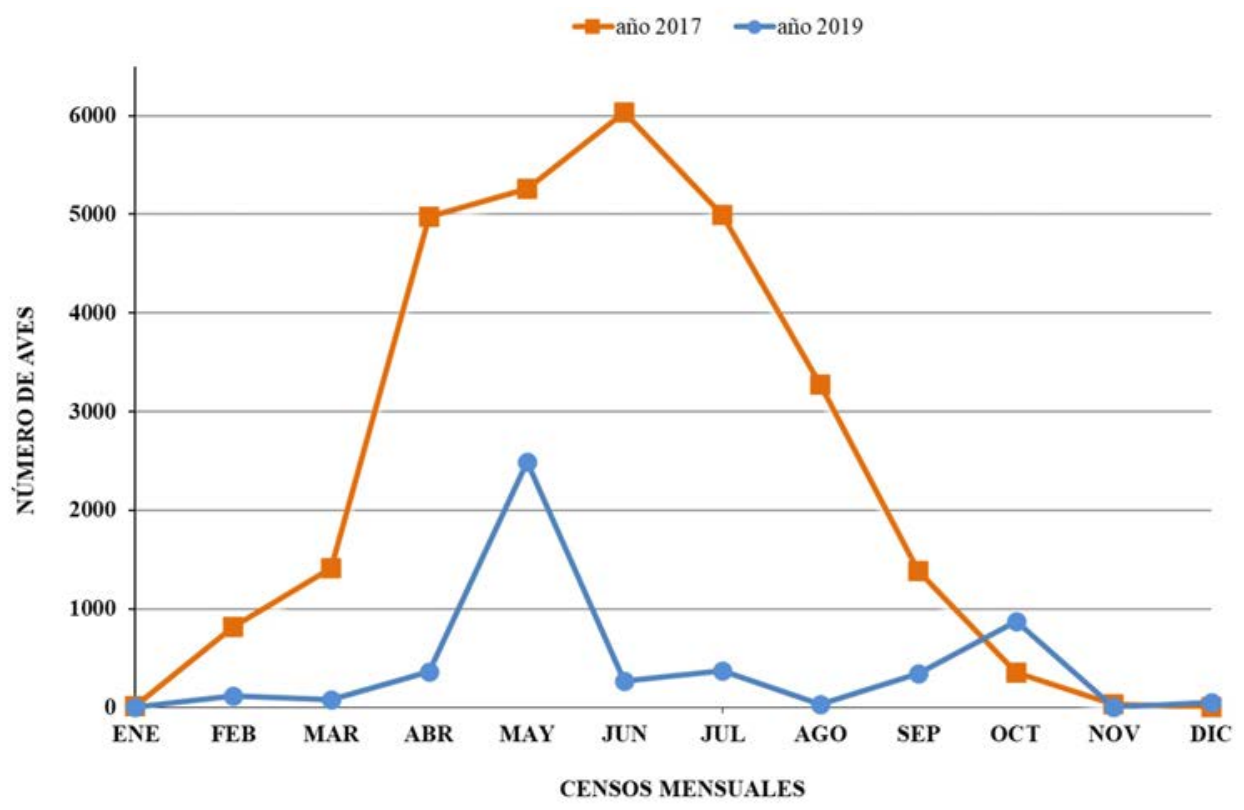

Figura 1.- Dinámica temporal (censos mensuales), de flamencos en el complejo lagunar de Pétrola-Corral Rubio-La Higuera. Años 2017 y 2019.

En la figura 2 se aprecia que el abandono de la colonia se produjo de forma importante hasta primeros de junio, y desde entonces de una forma más moderada. En las figuras 3 y 4 se aprecian sendos momentos del proceso de abandono. 


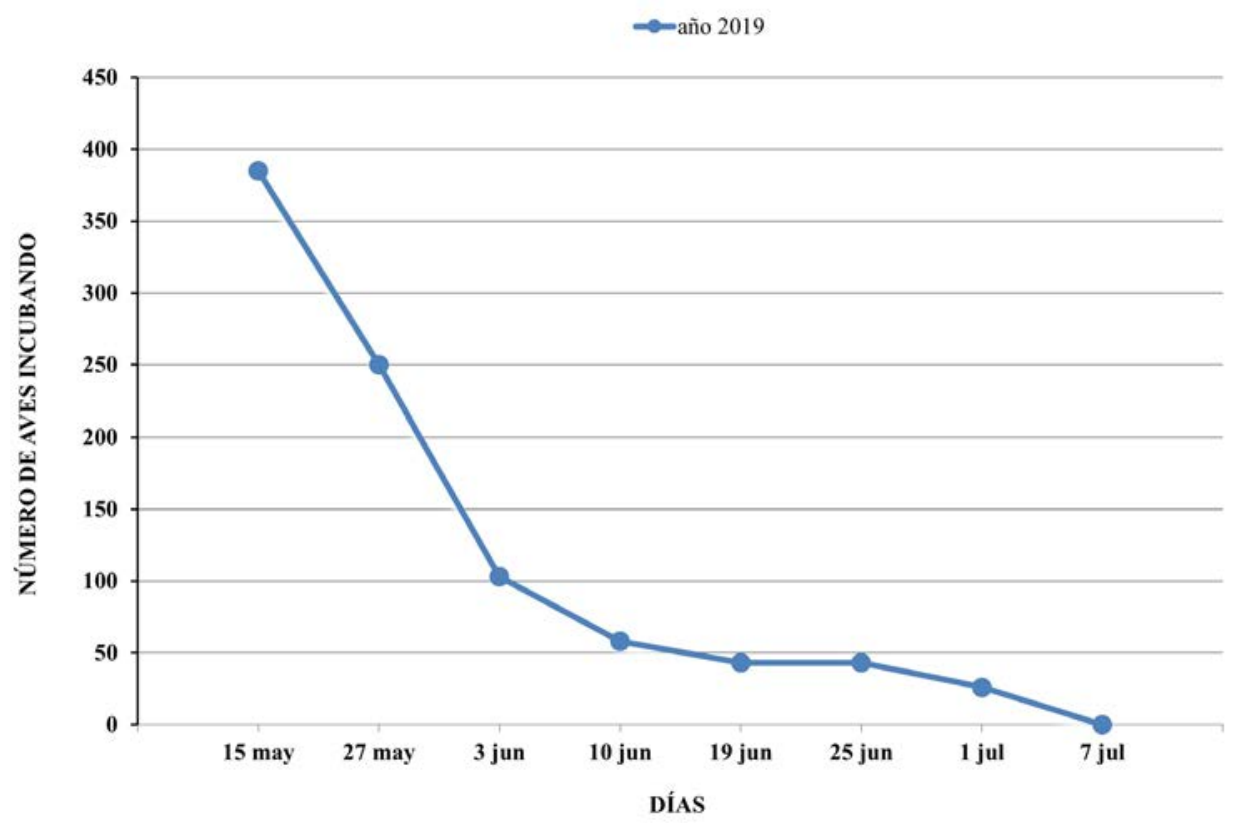

Figura 2.- Dinámica temporal en el número de aves incubando en la colonia de flamencos en la laguna de Pétrola, entre mediados de mayo y primeros de julio de 2019.

Los datos obtenidos entre 2017 y 2020 sobre la fenología de la reproducción del flamenco común en la laguna de Pétrola, concuerda con lo indicado sobre las 7 reproducciones anteriores (véase en Picazo, 2018), salvo el mencionado inicio tardío de la puesta en 2019.

La colonia se situó, durante los 3 periodos reproductores estudiados, en la isla mayor, que es la localización desde el año 2013. 


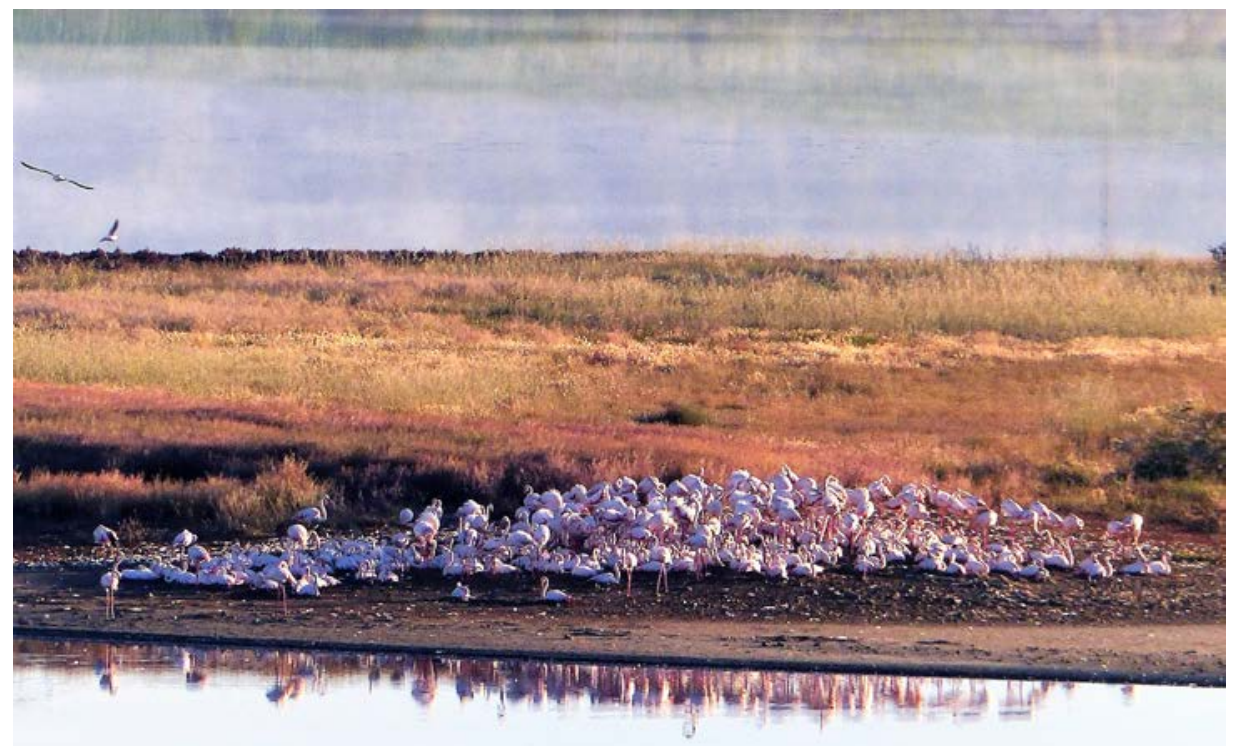

Figura 3.- Colonia de flamenco común en la laguna de Pétrola, el día 27/05/2019. Imagen: Juan Picazo.

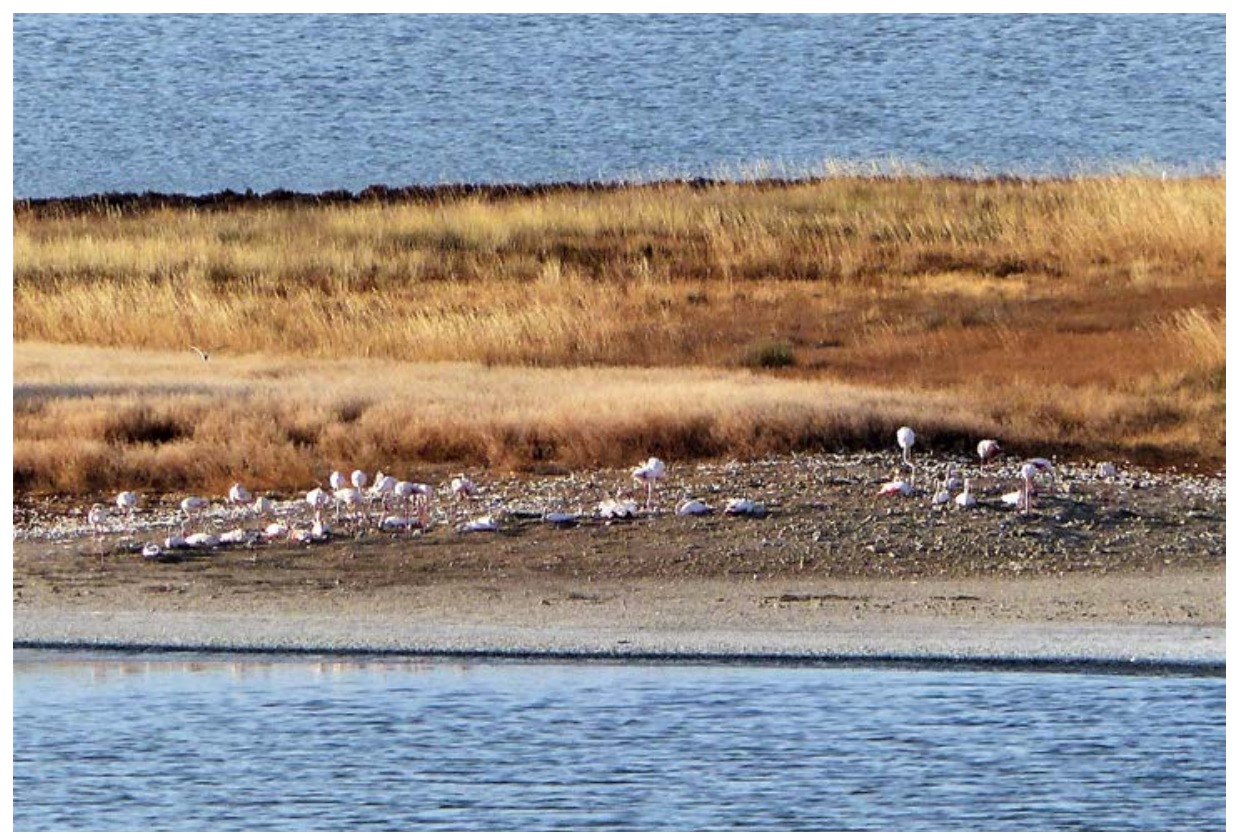

Figura 4.- Colonia de flamenco común en la laguna de Pétrola, el día 22/06/2019, donde se observa una gran cantidad de huevos abandonados. Imagen: Juan Picazo. 


\section{AGRADECIMIENTOS}

A quienes colaboraron en el seguimiento de la especie en Albacete durante el periodo 2017-2019, especialmente a José María García Jaén y Atilano Córcoles García.

José Antonio López Donate, director de la Reserva Natural de la laguna de Pétrola, nos facilitó el trabajo de campo gestionando las autorizaciones de la Junta de Comunidades de Castilla-La Mancha. José Fajardo realizó la traducción del Resumen al inglés.

Domingo Blanco y tres revisores anónimos más, mejoraron sustancialmente el original.

\section{BIBLIOGRAFÍA}

Johnson, A.R. y Cézilly, F. (2007). The Greater Flamingo. T. \& A.D. POYSER.

Picazo, J. (2011). Estatus, abundancia y distribución del Flamenco común (Phoenicopterus roseus) en la provincia de Albacete. Sabuco, 8: 187204. IEA.

Picazo, J. (2017a-j). Informe sobre el seguimiento del Flamenco común (Phoenicopterus roseus) en los humedales de Albacete. Febrero-septiembre de 2017. Informes inéditos.

Picazo, J. (2018). Datos sobre la reproducción del Flamenco común (Phoenicopterus roseus) en la laguna de Pétrola (Albacete, CastillaLa Mancha): periodo 1998-2016. En, Blanco, D.; Fajardo, J.; Ferrandis, P.; Gómez, J.; Picazo, J.; Sanz, D.; Valdés, A.; y Verde, A. (Coordinadores): III Jornadas sobre el Medio Natural Albacetense, pp. 152-160. Instituto de Estudios Albacetenses.

Picazo, J. (2019a-e). Informe sobre el seguimiento del Flamenco común (Phoenicopterus roseus) en los humedales de Albacete. Abril-agosto de 2019. Informes inéditos.

Picazo, J. (2019). Flamenco común. Phoenicopterus roseus. En, J. Picazo (Rec.): Prontuario de la Naturaleza Albacetense, pp. 145-165. Sabuco, 13: 152. 